\title{
Hedgehog pathway members Patched and Costal-2 exhibit differences in overgrowth autonomy in Drosophila melanogaster
}

\author{
Shannon L. Moore ${ }^{1}$, Frank C. Adamini ${ }^{1}$, Erik S. Coopes ${ }^{1}$, Dustin Godoy ${ }^{2}$, Shyra J. \\ Northington $^{1,3}$, Jordan M. Stewart ${ }^{1}$, Richard L. Tillet ${ }^{4}$, Kayla L. Bieser ${ }^{2}$, and Jacob D. Kagey ${ }^{\#}$
}

1. Biology Department, University of Detroit Mercy. Detroit, Michigan 48221.

2. Department of Physical and Life Sciences, Nevada State College. Henderson, Nevada 89002.

3. ReBUILDetroit, University of Detroit Mercy. Detroit, Michigan 48221.

4. Nevada Institute of Personalized Medicine, University of Nevada, Las Vegas, Nevada 89154.

\# Correspondence: kageyja@udmercy.edu 


\section{Abstract}

3 Genetic screens are used in Drosophila melanogaster to identify genes key in the regulation of 4 organismal development and growth. These screens have defined signaling pathways necessary 5 for tissue and organismal development which are evolutionarily conserved across species, 6 including Drosophila. Here we have used a Flp/FRT mosaic system to screen for conditional 7 regulators of cell growth and cell division in the Drosophila eye. The conditional nature of this 8 screen utilizes a block in the apoptotic pathway to prohibit the mosaic mutant cells from dying 9 via apoptosis. From this screen, we identified two different mutants that mapped to the

10 Hedgehog signaling pathway. Previously, we described a novel Ptc mutation and here we add to 11 the understanding of disrupting the Hh pathway with a novel allele of $\operatorname{Cos} 2$. Both of these Hh 12 components are negative regulators of the pathway, yet they depict mutant differences in the type 13 of overgrowth. Ptc mutations lead to overgrowth consisting of almost entirely wild type issue 14 (non-autonomous overgrowth), while the $\operatorname{Cos} 2$ mutation results in tissue that is overgrown in 15 both the mutant and wild type clones (both autonomous and non-autonomous). These differences 16 in tissue overgrowth are consistent in the Drosophila eye and wing. The observed difference is 17 correlated with a different pattern of deregulation of Mad, the downstream effector of DPP 18 signaling. This finding provides insight into pathway specific differences that may help to better 19 understand intricacies of developmental processes and human disease. 


\section{Introduction:}

Drosophila melanogaster is a long-established model system with particular usefulness in

22 understanding the genetic mechanisms underlying many life processes, including the genetic

23 regulation of growth and development. A large proportion of the genes that regulate growth in

24 developing tissues across species, including humans, were first identified using genetic screens

25 in Drosophila (Stone et al., 1996). The Flp/FRT genetic system has been used in genetic screens

26 to identify genes that are involved in regulating growth and differentiation by analyzing the

27 ratios of mutant to wild type tissue within the Drosophila eye. Genes identified in these screens

28 are frequently implicated in human diseases, and a number of these genes were initially

29 discovered in Drosophila and later found to be disease causing in human (Hariharan and Bilder,

30 2006; Harvey et al., 2003; Moberg et al., 2001; Wu et al., 2003).

31 In developing our genetic screen, we hypothesized that a subset of mutations would lead

32 to the removal of mutant cells through apoptosis (when homozygous), thus causing specific

33 mutantations to be missed in the initial iterations of the Flp/FRT screen (Golic and Lindquist,

34 1989; St Johnston, 2002). Since the inhibition of apoptosis remains a key step during

35 carcinogenesis for cancer cells to avoid a primary defense mechanism (Hanahan and Weinberg,

36 2000), this inhibition remains an important genetic process to understand from a disease

37 standpoint. Therefore, we conducted a second-generation conditional Flp/FRT EMS screen on

38 chromosome 2R in a genetic background blocking apoptosis, to identify conditional regulators of

39 cell growth and division (Kagey et al., 2012). A number of growth mutants conditional on a

40 block in apoptosis have been previously identified, including scribbled, providing further

41 rationale for the screen (Brumby and Richardson, 2003). 
From this screen we have previously reported on a number conditional regulators of cell

43 growth and cell division including $C P A$ and $S h n$, demonstrating the occurrence of these

44 conditional growth mutants (Bieser et al., 2019; Cosenza and Kagey, 2016). Here we describe

45 the nature of the over-growth caused by two of these mutants identified in the screen that are

46 both part of the Hedgehog signaling pathway, Patched (Ptc) and Costal-2 (Cos2).

The Hedgehog pathway (Hh) is a highly conserved signaling pathway essential to organismal development (Bellusci et al., 1997; Goodrich et al., 1996). This pathway was first

49 identified for its role in segment polarity during Drosophila embryo development (Nusslein-

50 Volhard and Wieschaus, 1980). Since that discovery, Hh has been found to be a critical pathway

51 in regulating cell differentiation and growth across model organisms. In humans, mutations in

52 the Hh pathway have been linked to both developmental abnormalities and disease later in life,

53 making it a relevant diagnostic and therapeutic target (Hahn et al., 1996).

The Hh pathway starts with the Hedgehog (Hh) ligand binding to the extracellular

55 receptor Patched (Ptc). When unbound, Ptc inhibits Smoothened (Smo). The binding of Hh stops

56 suppression of Smoothened, leading to downstream activation of proteins including Protein

57 Kinase A (PKA) and the kinesin Costal-2 (Cos2) (Jiang and Hui, 2008). When activated,

58 PKA/Cos2 cleave the transcriptional activator $\mathrm{Ci}$, allowing its translocation into the nucleus,

59 where it drives gene transcription (Wang and Holmgren, 2000). Ci has several known

60 transcriptional targets including Ptc, which then serves as a negative feedback loop for the

61 pathway (Xiong et al., 2015).

62 Previously, we reported on one mutant from the screen, Ptc ${ }^{B .2 .13}$, that serves as a

63 conditional regulator of overgrowth (Kagey et al., 2012). The Ptc ${ }^{B .2 .13}$ mutation to leads to non-

64 autonmous overgrowth driven by the non-autonomous activity of Yorkie and Mad in cells that 
65 are adjacent to the Ptc ${ }^{B .2 .13}$ clones. Complementing these findings was a companion study

66 utilizing the GMR-Hid mosaic screen conducted by the Bergmann lab (Fan and Bergmann,

67 2008).

68 Here, we add to the knowledge of the interplay between growth regulation, tissue

69 development, and cell survival in the Hh pathway. We find that Cos2 is also a Hh pathway

70 conditional regulator of cell growth, dependent upon a block in the canonical apoptosis pathway.

71 However, despite both Ptc and Cos2 serving as negative regulators of the Hh signaling pathway,

72 we find differences in the autonomous nature of the overgrowth: Ptc mutant clones drive a non-

73 autonomous overgrowth while $\operatorname{Cos} 2$ mutant clones lead to both an autonomous and non-

74 autonomous overgrowth. These findings provide insight into how disruptions to different points

75 of the Hh signaling pathway lead to different consequences for growth and development.

\section{Methods}

78 Conditional Genetic Screen for Regulators of Cell Growth and Division.

An EMS screen was conducted on chromosome 2R utilizing Flp/FRT system in the

81 mutations that disrupted cell division and growth, dependent on the Dark ${ }^{82}$ block of apoptosis

82 (Kagey et al., 2012). The $\operatorname{Dark}^{82}$ allele utilized has been shown to block developmental apoptosis

83 when homozygous (Akdemir et al., 2006). From the screen 137 mutants were identified and

84 stable stocks were created. Selected growth mutants were mapped via complementation mapping

85 to the Bloomington 2R Deficiency kit (Cook et al., 2012). Previously we mapped a novel allele

86 of Patched (Ptc ${ }^{B .2 .13}$ ) (Kagey et al., 2012). From the same screen here we report a novel allele of

87 Costal-2 $\left(\operatorname{Cos} 2^{F .1 .4}\right)$. The mutant stock $\operatorname{Cos} 2^{F .1 .4}$ failed to complement two previously 
88 characterized alleles of $\operatorname{Cos} 2, \operatorname{Cos} 2^{k 16101}$ and $\operatorname{Cos} 2^{H 29}$ (Christiansen et al., 2013; Roch et al.,

89 1998).

To identify the specific molecular lesion causing driving the growth phenotypes in Ptc

91 and Cos2 PCR primers were designed for the exons of each gene. DNA was isolated utilizing

$92 \mathrm{Li} / \mathrm{Cl}$, KAc from heterozygous $P t c^{B .2 .13}$ and $\operatorname{Cos} 2^{F .1 .4}$ animals. Following successful PCR

93 amplification, samples were sent for Sanger sequencing to identify heterozygous point mutations

94 in the chromatograms (Genewiz, New Jersey).

95

96 Genetics

97 In addition to the Dark $^{82}$ allele (Akdemir et al., 2006), the following genotypes were used

98 in these experiments Ey-Flp;FRT42D (BDSC), Ey-Flp; FRT42D, ubi-GFP, Ey-Flp; FRT42D,

99 M(2) (BDSC), UBX-Flp; FRT42D, ubi-GFP (BDSC), $\operatorname{Cos} 2^{H 29}$ allele (Christiansen et al., 2013),

100 and $\operatorname{Cos} 2^{\mathrm{K} 161010}$ (BDSC) (Roch et al., 1998). The 2R deficiency kit from the Bloomington

101 Drosophila Stock Center was used for genetic mapping via complementation tests (Cook et al.,

102 2012). All crosses were conducted at $25^{\circ} \mathrm{C}$.

103

104 Adult eye and wing visualization

105 Images were taken of the adult mosaic eyes at the same magnification (40x) under $70 \%$

106 ethanol to compare size of eye and ratio of mutant/wild type tissue. In cases where the cross was

107 setup to a stock containing the pigmented $\operatorname{Dark}^{82}$ allele, the 'Flp' stock contained an

108 unpigmented FRT42D chromosome. When the Dark $^{82}$ allele was not present the 'Flp' stock

109 utilized an FRT42D chromosome with pigmentation (FRT42D, ubi-GFP). Adult wings were

110 mounted to slides in vegetable oil and imaged at the same magnification (70x) to allow 
111 comparison of size differences between genotypes. Quantification of adult wing size was done

112 measuring pixels on Photoshop of at least 10 wings per genotype. For both wings and eyes the

113 images were taken on an AM Scope camera (AM scope 550 MA).

Immunohistochemistry

116 Imaginal eye and wing discs were dissected from wandering L3 larvae as previously

117 described (Kagey et al., 2012; Pellock et al., 2007). Briefly, imaginal discs were fixed in 4\%

118 paraformaldehyde and permeabilized in $0.3 \%$ PBST prior to staining with antibodies.

119 Antibodies from Developmental Studies Hybridoma Bank: anti-Ptc (mouse, 1:40), anti-Elav (rat

120 1:800), and anti-Ci (rat, 1:100) were used to stain eye and wing discs. Other antibodies used

121 were anti-phospho-Smad1/5 (pMad) (rabbit 1:100, Cell Signaling), anti-DIAP1 (mouse, 1:50)

122 (Yoo et al., 2002), and anti-GFP (chicken, 1:100, Aves Labs). Imaginal eye and wing discs were

123 visualized on a compound fluorescent microscope or confocal microscope using the Zen

124 microscopy software (Zeiss microscopy).

126 Analysis of fluorescent images

127 Imaginal eye and wing disc size and autonomy were calculated using Photoshop to

128 measure the number of pixels in each genotype. At least 10 imaginal discs were quantified for

129 each genotype. Autonomy was determined by calculating the percentage of non-GFP (mutant)

130 tissue divided by the whole tissue size using pixels in Photoshop. T-tests were used to analyze

131 the differences in autonomy between $P t c^{B .2 .13}$ and $\operatorname{Cos} 2^{F .1 .4}$ mosaic tissue, and $\mathrm{p}$ values were

132 calculated to determine the significance. For these comparisons, a p value $<0.05$ was used for

133 significance. To measure levels of pMad expression across the clonal boundaries florescent 
134 intensity was measured using the Zen microscopy line scan to measure signal intensity (Zeiss

135 microscopy).

\section{Results}

138 Ptc and Cos2 alleles were isolated from a conditional Flp/FRT eye screen and map to genes in

139 the Hedgehog pathway

140 In the genetic background of blocked apoptosis, mutants that disrupted cell growth and

141 cell division in the mosaic eye were isolated in an EMS Flp/FRT genetic screen (Kagey et al.,

142 2012). Previously, Ptc ${ }^{B .2 .13}$ was mapped as a novel allele of Ptc via complementation mapping

143 which resulted in a failure to complement the $P t c^{S 2}$ allele (Ingham et al., 1991; Kagey et al.,

144 2012).

Here we mapped the F.1.4 mutant using homozygous lethality and complementation tests

146 to the 2R Df kit (Cook et al., 2012). A region of failure to complement was identified in the

147 overlapping region between the deficiencies $D f(2 R) E D 1715$ and $D f(2 R) 1673$ from

148 2R:7,326,951..7,533,553. The F.1.4 mutant was mated to individual alleles within this region

149 and failed to complement two previously characterized $\operatorname{Cos} 2$ alleles, $\operatorname{Cos} 2^{k 16101}$ and $\operatorname{Cos} 2^{H 29}$,

150 indicating that $\operatorname{Cos}^{F .1 .4}$ is a novel allele of $\operatorname{Cos} 2$ (Christiansen et al., 2013; Roch et al., 1998).

151 To further confirm the location of these mutations and to understand the molecular

152 alterations, we conducted Sanger sequencing of both $P t c^{B .2 .13}$ and $\operatorname{Cos} 2^{F .1 .4}$ flies. For $P t c^{B .2 .13}$ and

$153 \operatorname{Cos} 2^{\text {F.1.4 }}$, a double-peak in each chromatogram causing missense mutations in an amino acid

154 conserved between flies and humans. In $P t c^{B .2 .13}$, a T-A at 2R:8,660,339 was identified resulting

155 in a Trp-173-Arg missense mutation (Supplemental Figure 1). In $\operatorname{Cos} 2^{\text {F.1.4 }}$, a T-A at

$1562 \mathrm{R}: 8,660,339$ was identified resulting in a Leu-951-Gln missense mutation (Supplemental Figure 
157 1). The identified missense mutations along with the failure to complement data from established

$158 P t c$ and $\operatorname{Cos}$ alleles establish $P t c^{B .2 .13}$ and $\operatorname{Cos} 2^{F .1 .4}$ as mutant alleles of the Hh pathway.

160 Ptc and Cos2 are conditional growth mutants necessary for eye development

162 mosaic eyes as compared to the control Dark $^{82}$ mosaic eyes (Figure 1A compared to 1B, mutant

163 tissue is pigmented) (Kagey et al., 2012). Here we add that $\operatorname{Cos}^{\text {F.1.4 }}$, Dark $^{82}$ also results in

164 dramatically overgrown mosaic eye when compared to the control mosaic Dark $^{82}$ (Figure 1A

165 compared to $1 \mathrm{C}$, mutant tissue pigmented). To visualize the ommatidial organization of the eye

166 we mated mutants to an FRT42D chromosome with GFP (Ey-Flp;FRT42D, ubi-GFP).

167 Fluorescent apotome images show the Dark $^{82}$ mosaic eye to have organized and patterned

168 ommatidial structure in both the $\operatorname{Dark}^{82} / \operatorname{Dark}^{82}$ clones (darker tissue) and adjacent wild type

169 clones (brighter GFP positive tissue) (Figure 1D). In contrast, both Dark $^{82}$, Ptc $^{B .2 .13}$ and Dark ${ }^{82}$,

$170 \operatorname{Cos} 2^{F .1 .4}$ have a disrupted ommatidial organization that can be visualized by the misalignment of

171 ommatidia throughout the eye, the disruption of ommatidial organization can be seen in both

172 mutant clones (darker tissue) and wild type clones (lighter tissue) suggesting that Hh pathway

173 disruption has both autonomous and non-autonomous effects on overall eye development (Figure

174 1E-F,).

175 To investigate the degree to which the block in apoptosis contributes to the overgrowth

176 phenotypes we reintroduced the wild type Dark allele through recombination, allowing

177 homozygous cells to again go through apoptosis in mosaic eyes. Without a block in apoptosis,

178 both $P t c^{B .2 .13}$ and $\operatorname{Cos} 2^{\text {F.1.4 }}$ mosaic eyes depict a partial rescue of overgrowth, indicating that

179 both mutant overgrowth phenotypes are, at least in part, dependent upon a block in apoptosis 
180 (Figure 1G-H, mutant tissue unpigmented). Though the eyes size is reduced when apoptosis is

181 reintroduced, eye organizational disruption remains and can be observed in both Hh mutants.

182 Additionally, neither $P t c^{B .2 .13}$ or $\operatorname{Cos} 2^{F .1 .4}$ mosaic eyes had any visible unpigmented tissue

183 (mutant tissue) cells which indicates that the majority (if not all) of the mutant cells may have

184 been cleared by apoptosis.

185 To look at eyes that are entirely comprised of mutant tissue we mated the Ptc ${ }^{B .2 .13}$ and

$186 \operatorname{Cos} 2^{\text {F.1.4 }}$ mutants to an FRT chromosome with a homozygous lethal mutation. In both cases

$187 P t c^{B .2 .13} \operatorname{Dark}^{82}$ and $\operatorname{Cos} 2^{\text {F.1.4 }}$ Dark $^{82}$ mutants resulted in pupal lethality and a severe reduction

188 in overall head size, highlighting the need for functional Hedgehog signaling in the developing

189 eye and overall organismal survival (Figure 1I-J).

191 Ptc and Cos2 mutations drive conditional overgrowth and pupal lethality in the mosaic wing

To investigate the generalized nature of this conditional Hh-deregulated overgrowth, we 193 analyzed mosaic wing discs phenotypes utilizing the $U B X-F l p$ driver. Previously, we found that

$194 P t c^{B .2 .13}$ Dark $^{82}$ mosaic wing led to a dramatic tissue overgrowth, resulting in pupal lethality due

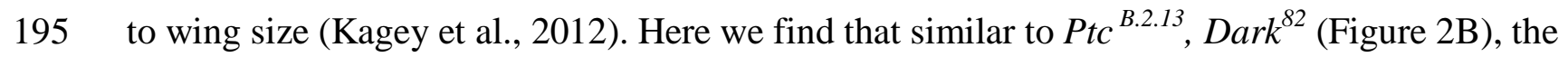

$196 \operatorname{Cos} 2^{F .1 .4}$, Dark $^{82}$ mosaic wing discs result in substantial tissue overgrowth and complete pupal

197 lethality (Figure 2A compared to 2C). While both the $P t c^{\text {B.2.13 }}$ Dark $^{82}$ and $\operatorname{Cos}^{\text {F.1.4 }}$, Dark $^{82}$

198 mosaic wing discs were substantially larger than the $\operatorname{Dark}^{82}$ control disc, we noted that the

199 overall size of the Ptc ${ }^{B .2 .13}$, Dark $^{82}$ mosaic disc was consistently larger than the $\operatorname{Cos} 2^{\text {F.1.4 }}$, Dark $^{82}$

200 mosaic wing disc, though the difference was not statistically significant $(\mathrm{p}=0.06355497)($ Figure

201 2D). Despite there not being a statistical difference in size, there was an observable biological

202 difference between $P t c^{B .2 .13}$ and $\operatorname{Cos} 2^{F .1 .4}$ mutants when the ability to undergo apoptosis was re- 
203 introduced by recombining the wild type $\operatorname{Dark}^{W T}$ allele. The reintroduction of apoptosis (via

$204 \operatorname{Dark}^{+}$) fully rescued the $\operatorname{Cos} 2^{\text {F.1.4 }}$ mosaic pupal lethality, while the majority of Ptc ${ }^{\text {B.2.13 }}$ mosaic

205 organisms still succumbed to pupal lethality (Figure 2E). Furthermore, the resulting $\operatorname{Cos} 2^{\text {F.1.4 }}$

206 mosaic wings were indistinguishable in size from control mosaic wings (Figure 2F). The rare

$207 P t c^{B .2 .13}$ escaper still had enlarged adults wing and depicted the 'wings held out' phenotype

208 (Figure 2G). So while both $P t c^{B .2 .13}$ and $\operatorname{Cos} 2^{F .1 .4}$ result in dramatic wing overgrowth there are

209 differences in the extent of tissue overgrowth based on which part of the Hh pathway is

210 dirsrupted.

Patched and Costal-2 exhibit differences in the autonomy of overgrowth

214 we observed differences in the ratio of mutant (pigmented) to wild type (unpigmented) tissue

215 (Figure1B, 1C). The Dark ${ }^{82}$, Ptc ${ }^{B .2 .13}$ mosaic eye is comprised of mostly wild-type tissue,

216 suggesting a non-autonomous overgrowth (Figure1B, 1E and (Kagey et al., 2012)), while the

$217 \operatorname{Dark}^{82}, \operatorname{Cos} 2^{F .1 .4}$ mosaic eye depicts more gross overgrowth (both autonomous and non-

218 autonomous tissue overgrowth Figure 1C, 1F). To measure the extent of this difference, we

219 utilized imaginal eye and wing discs to determine the percentage each disc is comprised of

220 homozygous mutant tissue in $P t c^{B .2 .13}$ and $\operatorname{Cos} 2^{F .1 .4}$ mutants. Using imaginal discs allowed for

221 quantification of 2-D tissue instead of 3-D eye ratios. In these imaginal discs we observe a

222 statistically significant difference $(\mathrm{p}=0.01827498)$ in which the Dark $^{82}$, Ptc $^{B .2 .13}$ eye discs are

223 comprised of $(8.1 \%)$ mutant tissue (non-GFP positive), while the Dark $^{82}$, Cos $2^{\text {F.1.4 }}$ eye discs are

224 comprised of (17.2\%) mutant tissue (non-GFP positive)(Figure 3A-C). This difference in

225 autonomous overgrowth despite both genotypes resulted in premature differentiation (and mitotic 
arrest) in the imaginal eye discs (observed by Elav expression in mutant clones before the

227 morphogenetic furrow, Supplemental Figure 2). Similarly, in the wing disc we find that $12.6 \%$

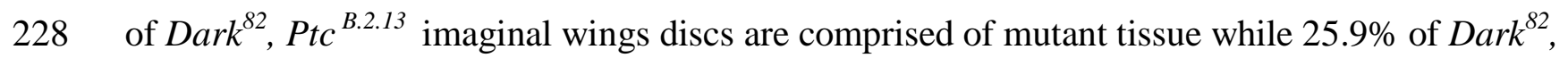

$229 \operatorname{Cos} 2^{\text {F.1.4 }}$ imaginal wing discs are comprised of mutant tissue, $\mathrm{p}=0.00605447$ (Figure 3D-F). As a

230 comparison, we have previously reported the Dark82 mosaic wing disc to be 38\%

$231 \operatorname{Dark}^{82} / \operatorname{Dark}^{82}$ mutant cells. The difference in tissue autonomy of Ptc and Cos 2 in the eye and

232 wing overgrowth autonomy suggest that these are pathway disruption differences and not a

233 phenomenon of tissue specific developmental signaling. To understand how mutations in the

234 same pathway could lead to different varieties of tissue overgrowth, we utilized imaginal disc

235 staining to observe molecular alterations that accompany these mutations.

Ptc and Cos2 autonomously de-regulate Hedgehog pathway signaling

Both Ptc and Cos2 are upstream negative regulators of the canonical Hedgehog signaling

239 pathway and function to sequester the transcription factor Cubitus interruptus (Ci) in the

240 cytoplasm (Aza-Blanc et al., 1997). Previously, we established that $P t c^{B .2 .13}$ mutant clones result

241 in an autonomous upregulation of Dpp, Patched, and Ci (Figure 4B, (Kagey et al., 2012)). Cos2

242 mutations have also been shown to autonomously upregulate downstream Hh targets

243 (Christiansen et al., 2012; Christiansen et al., 2013; Zadorozny et al., 2015). Here we support

244 these findings through the observation of an upregulation of cleaved $\mathrm{Ci}$ in Dark ${ }^{82}$, Ptc $^{\text {B.2.13 }}$ and

$245 \operatorname{Dark}^{82}, \operatorname{Cos} 2^{\text {F.1.4 }}$ imaginal wing disc clones (Figure 3B-C). Conversely, Dark ${ }^{82}$ mosaic wings

246 demonstrate a wild type expression pattern of $\mathrm{Ci}$ suggesting that the $\mathrm{Ci}$ overexpression is not

247 resultant from a block in apoptosis (Figure 3A). The deregulation of $\mathrm{Ci}$ in Ptc and Cos 2 mutants

248 occurs exclusively in clones on the anterior side of the wing disc correspondent with the 
249 expression domain of $\mathrm{Ci}$ (all wing discs are oriented with anterior to the left). Dark ${ }^{82}, \operatorname{Cos}^{\text {F.1.4 }}$

250 also phenocopies the Dark ${ }^{82}$, Ptc $^{B .2 .13}$ autonomous increase of Patched expression in the anterior

251 domain of the wing disc (Ptc is also a Ci target gene) (Supplemental Figure 3). Overall, this

252 suggests that mutations in Ptc or $\operatorname{Cos} 2$ mutations disrupt the Hh pathway and lead to an

253 autonomous increase of Hh signaling.

Ptc and Cos 2 mutant clones result in a non-autonomous up-regulation of DIAP1

Previously, our lab and others, have established a link between Hh deregulation and non-

257 autonomous DIAP1 up-regulation in both Ptc and Cos 2 mosaic tissue (Christiansen et al., 2012;

258 Christiansen et al., 2013; Kagey et al., 2012). To ensure this non-autonomous up regulation of

259 DIAP1 is consistent across Cos 2 alleles, we measured the levels of DIAP1 expression in mosaic

260 imaginal wing discs for $\operatorname{Cos} 2^{F .1 .4}$. As conveyed previously, $\operatorname{Dark}^{82}$, Ptc ${ }^{B .2 .13}$ leads to the non-

261 autonomous upreguation of DIAP1 (Figure 5A), which can be visualized as the halo of DIAP1

262 expression just outside of the $P t c^{B .2 .13}$ clonal borders. The Dark ${ }^{82}, \operatorname{Cos} 2^{F .1 .4}$ mutant also leads to

263 the non-autonomous increase in DIAP1 expression (Figure 5B) visualized as halos of DIAP1 up-

264 regulation immediately outside the $\operatorname{Cos} 2^{F .1 .4}$ clones. To more easily visualize the non-

265 autonomous nature of the DIAP1 overexpression we utilized Ci to mark the mutant clones which

266 provides an easier observation of expression at the clonal boundaries. Further, this up-regulation

267 of DIAP1 is confined to the anterior compartment suggesting it is dependent on the $\mathrm{Ci}$

268 overexpression autonomously to facilitate the non-autonomous DIAP1 up-regulation (Figure 5). 
272 tissue proliferation of the $\operatorname{Dark}^{82}, P t c^{B .2 .13}$ mutants were shown to be dependent on the

273 concurrent non-autonomous up regulation of $\mathrm{pMad} 1 / 5$ and the interaction of pMad and Yorkie to

274 drive non-autonomous survival and proliferation (Kagey et al., 2012). Here we investigated if

$275 \operatorname{Cos} 2^{F .1 .4}$ mutant clones also de-regulate pMad in a similar manner. The non-autonomous

276 activation of pMad1/5 in $\operatorname{Dark}^{82}$, Ptc $^{B .2 .13}$ mosaic wing discs, is visualized by the demonstrable

277 increase in pMad expression just outside of the mutant clone (mutant clones again marked by $\mathrm{Ci}$

278 expression to visualize pMad expression at the clonal boundaries) (Figure 6A-A'’). This non-

279 autonomous increase is also seen via line scan, where the peaks of pMad expression are just

280 outside of the mutant clonal boundaries and dissipate further away from the mutant clone (Figure

2816 6, clones marked by arrows).

expression. However, in contrast to the purely non-autonomous expression seen in the Ptc ${ }^{\text {B.2.13 }}$

284 clones, in $\operatorname{Cos} 2^{F .1 .4}$ the up-regulation of pMad is both autonomous (within the clone) and non-

285 autonomous (adjacent to the clone) (Figure 6C'-C', mutant clones marked by Ci to visualize

286 boundary expression). We again used a line scan to visualize the pMad expression intensity. The

287 high levels of pMad expression came from within the mutant clone and then remained high

288 outside of the clonal boundary (Figure 6D, clonal boundaries arrows). This indicates that while

289 both Ptc and $\operatorname{Cos} 2$ mutant tissue lead to a non-autonomous increase of pMad, only $\operatorname{Cos} 2$ has an

290 autonomous upregulation of pMad expression. The regions which pMad overexpression is

291 observed correlate to the nature of tissue overgrowth, non-autonomous, for Ptc ${ }^{B .2 .13}$ and both

292 autonomous and non-autonomous for $\operatorname{Cos} 2^{\text {F.1.4. }}$. 


\section{Discussion}

\section{Overview}

296 We designed a genetic screen to identify negative regulators of cell growth and cell

297 division that were conditional on a need for a block in apoptosis. From this screen, we isolated

298 and mapped a novel allele of $\operatorname{Cos} 2, \operatorname{Cos} 2^{F .1 .4}$, as a conditional growth regulator from an EMS

299 Flp/FRT mosaic eye screen. This finding builds upon previous data where we identified another

300 member of the hedgehog signaling pathway, $P t c^{B .2 .13}$, in the same screen, providing confirmation

301 that disruption in the hedgehog pathway can lead to conditional tissue overgrowth in Drosophila

302 (Kagey et al., 2012). While both Ptc and Cos2 mutants result in dramatic eye and wing

303 overgrowth due to the autonomous disruption of the Hh pathway, we observed that the nature of

304 the overgrowth differs between Ptc and Cos 2 mutants. Ptc mosaic tissue displays a distinct non-

305 autonomous overgrowth whereas $\operatorname{Cos} 2$ mosaic tissue results in both autonomous and non-

306 autonomous overgrowth. These differences were consistent in both the eye and wing imaginal

307 discs. Our data indicate that this difference may be due to the differential pattern of pMad

308 deregulation. The up-regulation of pMad has previously been demonstrated to drive tissue

309 overgrowth (Kagey et al., 2012; Oh and Irvine, 2011), and the pattern of pMad deregulation in

310 Ptc and $\operatorname{Cos} 2$ mosaic tissue directly matches the patterns of overgrowth.

312 Association of cell survival and Hedgehog signaling

313 Our results suggest that a functional Hh signaling pathway is necessary for cell survival

314 in imaginal disc development. Mutations to the Ptc and Cos 2 members of the hedgehog pathway

315 led to cell death. When apoptosis was blocked, Ptc and Cos 2 mutants depicted dramatic 
overgrowth, which was rescued in both the eye and wing when the wild type Dark allele was

317 reintroduced, thus reinstating cell death.

This is further supported by the findings of the Bergman lab that identified multiple

319 members of the Hh pathway in the GMR-Hid Flp/FRT screen (Christiansen et al., 2012;

320 Christiansen et al., 2013; Fan and Bergmann, 2008). This study also observed an autonomous

321 increase in cell death when the Hh pathway is disrupted. The finding that this cell death is

322 accompanied by a concurrent non-autonomous survival signal may be due to the compensatory

323 proliferation resulting from the loss of Hh-dependent cell death (Worley et al., 2012). This

324 concurrent non-autonomous DIAP1 and proliferation increase could be a mechanism of tissue

325 regeneration from which dying cells signal to their neighbors to proliferate. In our scenario, we

326 have removed the ability of the Hh deficient cells to die through the canonical apoptotic

327 pathway, therefore creating a constitutive non-autonomous survival and growth signaling leading

328 to eye and wing overgrowth.

Ptc and Cos2 exhibit different patterns of pMad deregulation

332 (including $\mathrm{Ci}$, and Ptc); however, there may be a difference in which type of cells can interpret

333 the increased Hh target Dpp. Given that Dpp is a morphogen, both autonomous and non-

334 autonomous cells would receive the Dpp signal via the receptors Thick Veins and Punt

335 (Hamaratoglu et al., 2014). However, as we previously demonstrated, the Ptc ${ }^{B .2 .13}$ mutant clones

336 also autonomously upregulated the inhibitory smad, Daughters Against Decapentaplegic (Dad)

337 (Goldstein et al., 2011; Kagey et al., 2012). We hypothesize that a lack of Dad upregulation in

338 the $\operatorname{Cos} 2^{F .1 .4}$ mutant clones would result in an autonomous increase proliferation due to the Dpp 
mediated pMad activation (as seen in Figure 6). Alternatively, it is possible that a differential

340 downregulation of Tkv downstream of the Hh signaling could be leading to the differential

341 activation of pMad between Ptc and Cos2 mutant clones (Tanimoto et al., 2000). In either

342 scenario, the difference in autonomous pMad upregulation between Ptc and $\operatorname{Cos} 2$ mosaic tissue

343 highlights the importance of understanding how disrupting different points of conserved

344 pathways impacts tissue patterning and growth.

A detailed understanding of the precise genetic mechanisms and pathways leading to de-

348 regulated growth in Hh mutations will be important in developing diagnostic and treatment tools

349 to address Hh de-regulated tumors in humas. Hedgehog pathway mutations are associated with

350 various types of human cancers, including medulloblastoma; basal cell carcinoma; glioma; and

351 breast, colorectal, pancreatic, and prostate cancer (Jiang and Hui, 2008; Skoda et al., 2018). The

352 loss of Ptc also increases the occurrence of these types of tumors in mice, providing additional

353 models for study (Wu et al., 2011). There is evidence of both Ptc and Kif7 (the human homolog

354 of Cos2) being down-regulated in different human cancers, including basal cell carcinoma and

355 ovarian cancer (Yao et al., 2019). Fully understanding the phenotypic differences that arise from

356 disruptions at different points in the Hh pathway may ultimately help with personalized medicine

357 and treatment decisions for patients with Hh-deregulated tumors.

\section{Acknowledgements}

360 We thank A. Bergmann, T. Cook, and K. Moberg for Drosophila stocks and reagents. We thank

361 T. Cook and E. Kagey for helpful comments on earlier drafts of this manuscript. Antibodies were 
362 obtained from the Developmental Studies Hybridoma Bank, created by the NICHD of the NIH

363 and maintained at The University of Iowa, Department of Biology, Iowa City, IA 52242. Stocks

364 obtained from the Bloomington Drosophila Stock Center (NIH P40OD018537) were used in this

365 study.

366

\section{Competing Interests}

$368 \quad$ No competing interests are declared.

369

$370 \quad$ Funding

371 This work was supported by the National Science Foundation (2021146 to KLB and

372 JDK); and the National Institutes of Health BUILD initiative (TL4GM118983 to SJN).

373

374

375 


\section{Reference:}

377

378

379

380

381

382

383

384

385

386

387

388

389

390

391

392

393

394

395

396

397

398

399

400

401

402

403

404

405

406

407

408

409

410

411

412

413

414

415

416

417

418

419

420

421

422

423

424

425

Akdemir, F., Farkas, R., Chen, P., Juhasz, G., Medved'ova, L., Sass, M., Wang, L., Wang, X., Chittaranjan, S., Gorski, S.M., Rodriguez, A. and Abrams, J.M., 2006. Autophagy occurs upstream or parallel to the apoptosome during histolytic cell death. Development. 133, 1457-65.

Aza-Blanc, P., Ramirez-Weber, F.A., Laget, M.P., Schwartz, C. and Kornberg, T.B., 1997. Proteolysis that is inhibited by hedgehog targets Cubitus interruptus protein to the nucleus and converts it to a repressor. Cell. 89, 1043-53.

Bellusci, S., Furuta, Y., Rush, M.G., Henderson, R., Winnier, G. and Hogan, B.L., 1997. Involvement of Sonic hedgehog (Shh) in mouse embryonic lung growth and morphogenesis. Development. 124, 53-63.

Bieser, K., Sanford, J., Saville, K., Arreola, K., Ayres, Z., Basulto, D., Benito, S., Breen, C., Brix, J., Brown, N., Burton, K., Chadwick, T., Chen, M., Chu, K., Corbett, B., Dill, Z., Faughender, M., Hickey, A., Julia, J., Kelty, S., Jobs, B., Krason, B., Lam, B., McCullough, C., McEwen, B., McKenzie, J., McQuinn, K., Moritz, C., Myers, K., Naugle, E., Nutter, A., O'Conke, D., O'Grondik, M., Patel, K., Rudowski, S., Sberna, E., Stall, G., Steiner, T., Tanriverdi, E., Torres Patarroyo, N., Traster, V., Tsai, L., Valenti, A., Villegas, M., Voors, S., Watson, K., Wright, M. and Kagey, J., 2019. Genetic mapping of shn(E.3.2) in Drosophila melanogaster. MicroPubl Biol. 2019.

Brumby, A.M. and Richardson, H.E., 2003. scribble mutants cooperate with oncogenic Ras or Notch to cause neoplastic overgrowth in Drosophila. EMBO J. 22, 5769-79.

Christiansen, A.E., Ding, T. and Bergmann, A., 2012. Ligand-independent activation of the Hedgehog pathway displays non-cell autonomous proliferation during eye development in Drosophila. Mech Dev. 129, 98-108.

Christiansen, A.E., Ding, T., Fan, Y., Graves, H.K., Herz, H.M., Lindblad, J.L. and Bergmann, A., 2013. Non-cell autonomous control of apoptosis by ligand-independent Hedgehog signaling in Drosophila. Cell Death Differ. 20, 302-11.

Cook, R.K., Christensen, S.J., Deal, J.A., Coburn, R.A., Deal, M.E., Gresens, J.M., Kaufman, T.C. and Cook, K.R., 2012. The generation of chromosomal deletions to provide extensive coverage and subdivision of the Drosophila melanogaster genome. Genome Biol. 13, R21.

Cosenza, A. and Kagey, J.D., 2016. The Mapping and Characterization of Cruella (Cru), a Novel Allele of Capping Protein a $(\mathrm{Cpa})$, Identified from a Conditional Screen for Negative Regulators of Cell Growth and Cell Division. Advances in Bioscience and Biotechnology 7, 373-380.

Fan, Y. and Bergmann, A., 2008. Apoptosis-induced compensatory proliferation. The Cell is dead. Long live the Cell! Trends Cell Biol. 18, 467-73.

Goldstein, J.A., Kelly, S.M., LoPresti, P.P., Heydemann, A., Earley, J.U., Ferguson, E.L., Wolf, M.J. and McNally, E.M., 2011. SMAD signaling drives heart and muscle dysfunction in a Drosophila model of muscular dystrophy. Hum Mol Genet. 20, 894-904.

Golic, K.G. and Lindquist, S., 1989. The FLP recombinase of yeast catalyzes site-specific recombination in the Drosophila genome. Cell. 59, 499-509.

Goodrich, L.V., Johnson, R.L., Milenkovic, L., McMahon, J.A. and Scott, M.P., 1996. Conservation of the hedgehog/patched signaling pathway from flies to mice: induction of a mouse patched gene by Hedgehog. Genes Dev. 10, 301-12.

Hahn, H., Wicking, C., Zaphiropoulous, P.G., Gailani, M.R., Shanley, S., Chidambaram, A., Vorechovsky, I., Holmberg, E., Unden, A.B., Gillies, S., Negus, K., Smyth, I., Pressman, C., Leffell, D.J., Gerrard, B., Goldstein, A.M., Dean, M., Toftgard, R., Chenevix-Trench, G., Wainwright, B. and Bale, A.E., 1996. Mutations of the human homolog of Drosophila patched in the nevoid basal cell carcinoma syndrome. Cell. 85, 841-51. 
426

427

428

429

430

431

432

433

434

435

436

437

438

439

440

441

442

443

444

445

446

447

448

449

450

451

452

453

454

455

456

457

458

459

460

461

462

463

464

465

466

467

468

469

470

471

472

473

474

475

476

Hamaratoglu, F., Affolter, M. and Pyrowolakis, G., 2014. Dpp/BMP signaling in flies: from molecules to biology. Semin Cell Dev Biol. 32, 128-36.

Hanahan, D. and Weinberg, R.A., 2000. The hallmarks of cancer. Cell. 100, 57-70.

Hariharan, I.K. and Bilder, D., 2006. Regulation of imaginal disc growth by tumor-suppressor genes in Drosophila. Annu Rev Genet. 40, 335-61.

Harvey, K.F., Pfleger, C.M. and Hariharan, I.K., 2003. The Drosophila Mst ortholog, hippo, restricts growth and cell proliferation and promotes apoptosis. Cell. 114, 457-67.

Ingham, P.W., Taylor, A.M. and Nakano, Y., 1991. Role of the Drosophila patched gene in positional signalling. Nature. 353, 184-7.

Jiang, J. and Hui, C.C., 2008. Hedgehog signaling in development and cancer. Dev Cell. 15, 801-12.

Kagey, J.D., Brown, J.A. and Moberg, K.H., 2012. Regulation of Yorkie activity in Drosophila imaginal discs by the Hedgehog receptor gene patched. Mech Dev. 129, 339-49.

Moberg, K.H., Bell, D.W., Wahrer, D.C., Haber, D.A. and Hariharan, I.K., 2001. Archipelago regulates Cyclin E levels in Drosophila and is mutated in human cancer cell lines. Nature. 413, 311-6.

Nusslein-Volhard, C. and Wieschaus, E., 1980. Mutations affecting segment number and polarity in Drosophila. Nature. 287, 795-801.

Oh, H. and Irvine, K.D., 2011. Cooperative regulation of growth by Yorkie and Mad through bantam. Dev Cell. 20, 109-22.

Pellock, B.J., Buff, E., White, K. and Hariharan, I.K., 2007. The Drosophila tumor suppressors Expanded and Merlin differentially regulate cell cycle exit, apoptosis, and Wingless signaling. Dev Biol. 304, 102-15.

Roch, F., Serras, F., Cifuentes, F.J., Corominas, M., Alsina, B., Amoros, M., Lopez-Varea, A., Hernandez, R., Guerra, D., Cavicchi, S., Baguna, J. and Garcia-Bellido, A., 1998. Screening of larval/pupal P-element induced lethals on the second chromosome in Drosophila melanogaster: clonal analysis and morphology of imaginal discs. Mol Gen Genet. 257, 103-12.

Skoda, A.M., Simovic, D., Karin, V., Kardum, V., Vranic, S. and Serman, L., 2018. The role of the Hedgehog signaling pathway in cancer: A comprehensive review. Bosn J Basic Med Sci. 18, 8-20.

St Johnston, D., 2002. The art and design of genetic screens: Drosophila melanogaster. Nat Rev Genet. 3, 176-88.

Stone, D.M., Hynes, M., Armanini, M., Swanson, T.A., Gu, Q., Johnson, R.L., Scott, M.P., Pennica, D., Goddard, A., Phillips, H., Noll, M., Hooper, J.E., de Sauvage, F. and Rosenthal, A., 1996. The tumour-suppressor gene patched encodes a candidate receptor for Sonic hedgehog. Nature. 384, 129-34.

Tanimoto, H., Itoh, S., ten Dijke, P. and Tabata, T., 2000. Hedgehog creates a gradient of DPP activity in Drosophila wing imaginal discs. Mol Cell. 5, 59-71.

Wang, Q.T. and Holmgren, R.A., 2000. Nuclear import of cubitus interruptus is regulated by hedgehog via a mechanism distinct from $\mathrm{Ci}$ stabilization and $\mathrm{Ci}$ activation. Development. 127, 3131-9.

Worley, M.I., Setiawan, L. and Hariharan, I.K., 2012. Regeneration and transdetermination in Drosophila imaginal discs. Annu Rev Genet. 46, 289-310.

Wu, S., Huang, J., Dong, J. and Pan, D., 2003. hippo encodes a Ste-20 family protein kinase that restricts cell proliferation and promotes apoptosis in conjunction with salvador and warts. Cell. 114, 445-56.

Wu, X., Northcott, P.A., Croul, S. and Taylor, M.D., 2011. Mouse models of medulloblastoma. Chin J Cancer. 30, 442-9.

Xiong, Y., Liu, C. and Zhao, Y., 2015. Decoding Ci: from partial degradation to inhibition. Dev Growth Differ. 57, 98-108. 

of KIF7 indicates poor prognosis in epithelial ovarian cancer. Cancer Biomark. 26, 481489. H.A. and Hay, B.A., 2002. Hid, Rpr and Grim negatively regulate DIAP1 levels through distinct mechanisms. Nat Cell Biol. 4, 416-24. interactions to Hedgehog signaling in Drosophila. Development. 142, 931-42. 


\section{Figure 1}
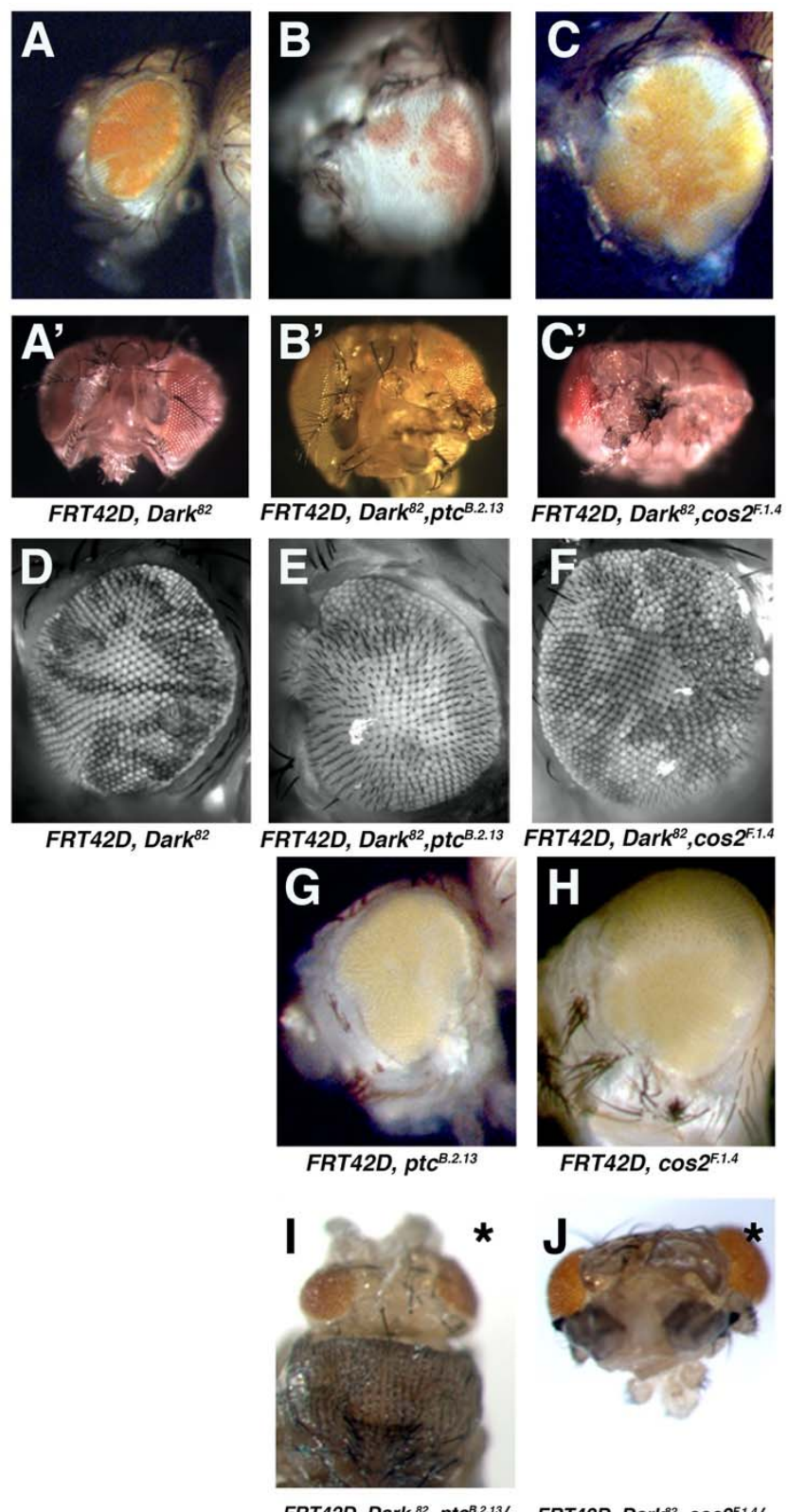

FRT42D, $\cos 2^{F 1.4}$

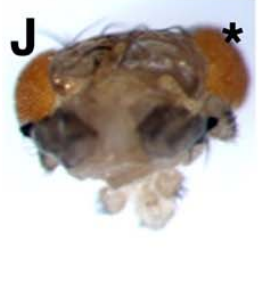

$M(2)$

FRT42D, Dark ${ }^{82}, \cos ^{5.1 .4 /}$ $M(2)$

Figure 1: Alleles of Patched and Costal-2 isolated from the same conditional Flp/FRT screen as negative regulators of cell growth and cell division in the eye.

Mosaic adult eyes imaged via light microscopy by mating Ey-Flp;FRT42D to (A) control eye $F R T 42 D$, Dark ${ }^{82}$ (B) ;FRT42D, ptc $^{B .2 .13}, \operatorname{Dark}^{82}$ (C) FRT42D, $\operatorname{Cos}^{F .1 .4}, \operatorname{Dark}^{82}$ (mutant tissue pigmented $m w+$ ). Dorsal images of each genotype showed in A'-C'. Fluorescent apotome images to visualize ommatidial organization by mating $E y$ Flp;FRT42D, ubi $>$ GFP to (D) control eye ;FRT42D, $\operatorname{Dark}^{82}$ (E) ;FRT42D, $\operatorname{ptc}^{\text {B.2.13 }}, \operatorname{Dark}^{82}$ (F) ;FRT42D, $\operatorname{Cos}^{F .1 .4}$, $\operatorname{Dark}^{82}$ (mutant tissue, darker, fluorescent negative). Light microscope images without Dark $^{82}$ allele through mating Ey-Flp;FRT42D, ubi-GFP to $(\mathrm{G}) ; F R T 42 D, p t c^{B .2 .13}(\mathrm{H}) ; F R T 42 D, \operatorname{Cos} 2^{F .1 .4}$ (mutant tissue is non-pigmented). Light microscope images of heads comprised entirely of mutant tissue by mating Ey-Flp;FRT42D, M(2) to (I) FRT42D, $P t c^{B .2 .13}, \operatorname{Dark}^{82}(\mathrm{~J}) ; F R T 42 D, \operatorname{Cos} 2^{F .1 .4}, \operatorname{Dark}^{82}$ (mutant tissue is pigmented $m w+$ ). * denotes pupal lethality 


\section{Figure 2}
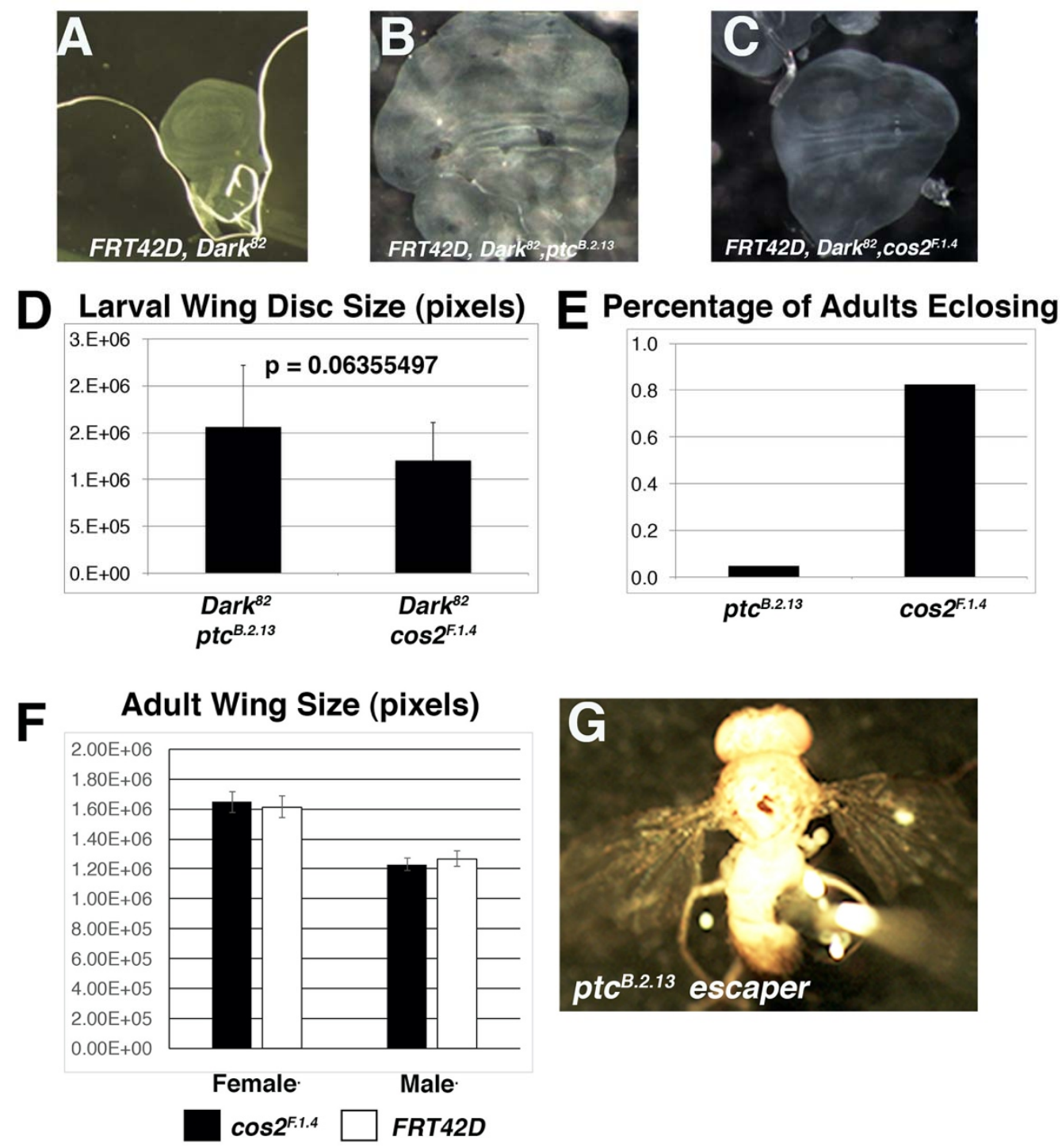

Figure 2: $P t c^{B .2 .13}$ and $\operatorname{Cos} 2^{F .1 .4}$ exhibit conditional overgrowth phenotypes in the Drosophila wing that result in pupal lethality.

Third instar imaginal wing discs were visualized by light microscopy for UBX-Flp:FRT42D mated to (A) control eye FRT42D, $\operatorname{Dark}^{82}$ (B) FRT42D, ptc $^{B .2 .13}$, $\operatorname{Dark}^{82}$ (C) FRT42D, $\operatorname{Cos}^{\text {F.1.4 }}$, Dark $^{82}$. Both Ptc and Cos2 wing discs resulted in complete pupal lethality. Average size of third instar imaginal wing disc (in pixels) for (D) FRT42D, $p t c^{B .2 .13}$, Dark $^{82}$ and FRT42D, $\operatorname{Cos}^{\text {F.1.4 }}$, Dark ${ }^{82}$ mosaic wing discs. Error bars represent standard deviation.

Difference is not statistically significant. (E) Percentage of adults that eclosed for without Dark ${ }^{82}$ allele for FRT42D, $p t c^{B .2 .13}$ and FRT42D, $\operatorname{Cos} 2^{F .1 .4}$ (n=100 for each genotype). (F) Comparison of adult wing size (in pixels) of control $(F R T 42 D)$ and $\left(F R T 42 D, \operatorname{Cos} 2^{F .1 .4}\right)$ wings, 10 wings per genotype and sex. (G) Image of $F R T 42 D$, ptcB.2.13 escaper, depicting wings held out phenotype. 


\section{Figure 3}
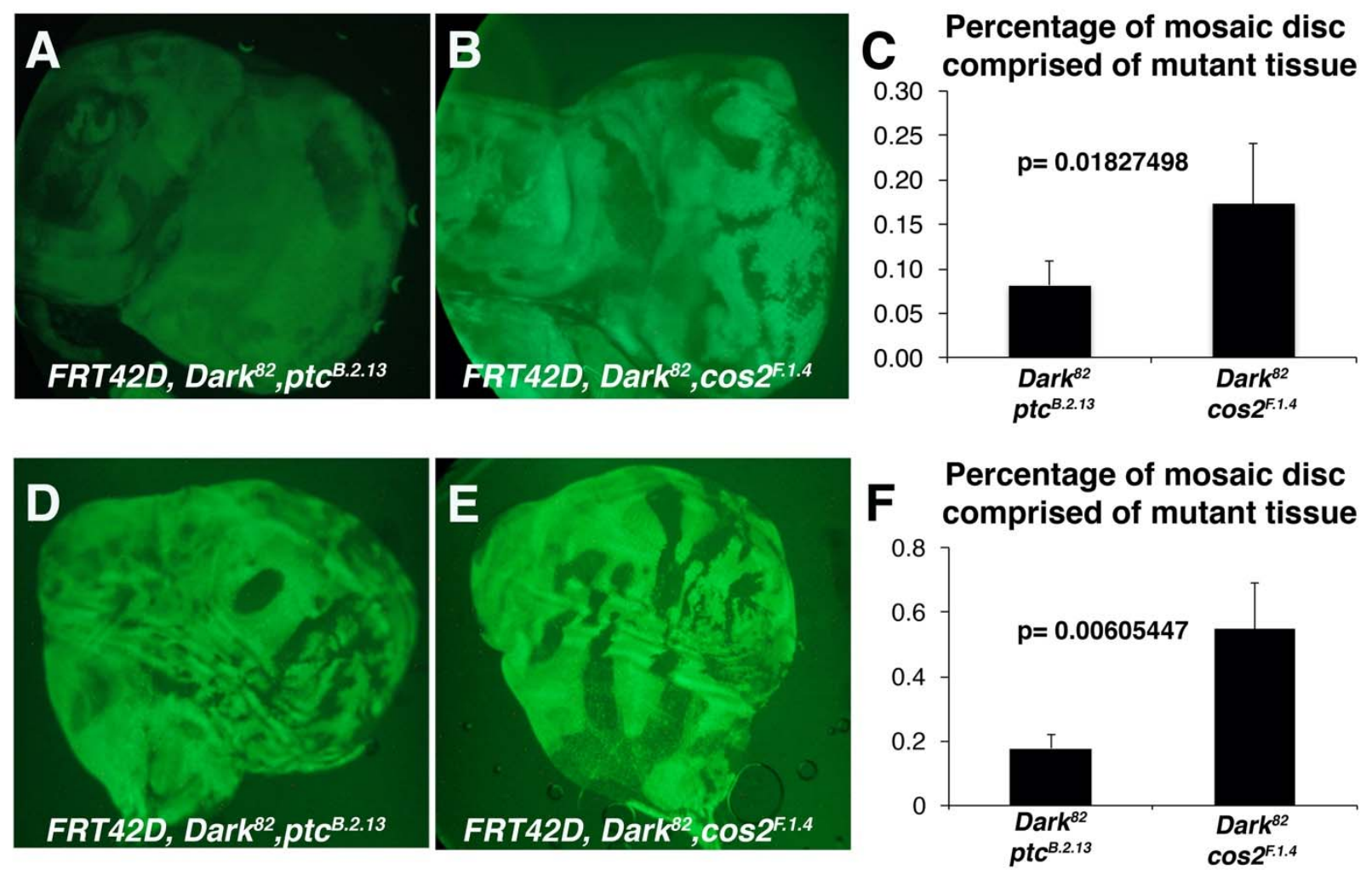

Figure 3: $P t c^{B .2 .13}$ and $\operatorname{Cos}^{F .1 .4}$ exhibit distinct types of autonomous overgrowth in the eye and wing.

Third instar imaginal eye discs were visualized by fluorescent microscopy for crosses of Ey-Flp:FRT42D, ubi-GFP mated to (A) FRT42D, ptc ${ }^{B .2 .13}, \operatorname{Dark}^{82}$ (B) FRT42D, $\operatorname{Cos}^{F .1 .4}$ Dark $^{82}$ (homozygous mutant tissue is GFP negative). (C) Percentage of mosaic disc comprised of mutant tissue for FRT42D, $p t c^{B .2 .13}, \operatorname{Dark}^{82}$ and FRT42D, $\operatorname{Cos} 2^{F .1 .4}$, Dark $^{82}$ measured by pixels. $>10$ imaginal discs quantified for each genotype. Error bars represent standard deviation and $\mathrm{p}$ value is from paired T-test. Third instar imaginal wing discs were visualized by light microscopy for crosses of UBX-Flp:FRT42D, ubi-GFP mated to (D) FRT42D, ptc ${ }^{\text {B.2.13 }}$, $\operatorname{Dark}^{82}$ (E) FRT42D, Cos $2^{F .1 .4}$, Dark ${ }^{82}$ (homozygous mutant tissue is GFP negative). (F) Percentage of mosaic disc comprised of mutant tissue for $F R T 42 D$, ptc ${ }^{B .2 .13}$, $\operatorname{Dark}^{82}$ and FRT42D, $\operatorname{Cos} 2^{F .1 .4}$, Dark ${ }^{82}$ measured by pixels. $>10$ imaginal discs quantified for each genotype. Error bars represent standard deviation and $\mathrm{p}$ value shown from paired T-test. 
bioRxiv preprint doi: https://doi.org/10.1101/2021.04.09.439246; this version posted April 11, 2021. The copyright holder for this preprint (which was not certified by peer review) is the author/funder, who has granted bioRxiv a license to display the preprint in perpetuity. It is made available under aCC-BY-NC-ND 4.0 International license.

\section{Figure 4}
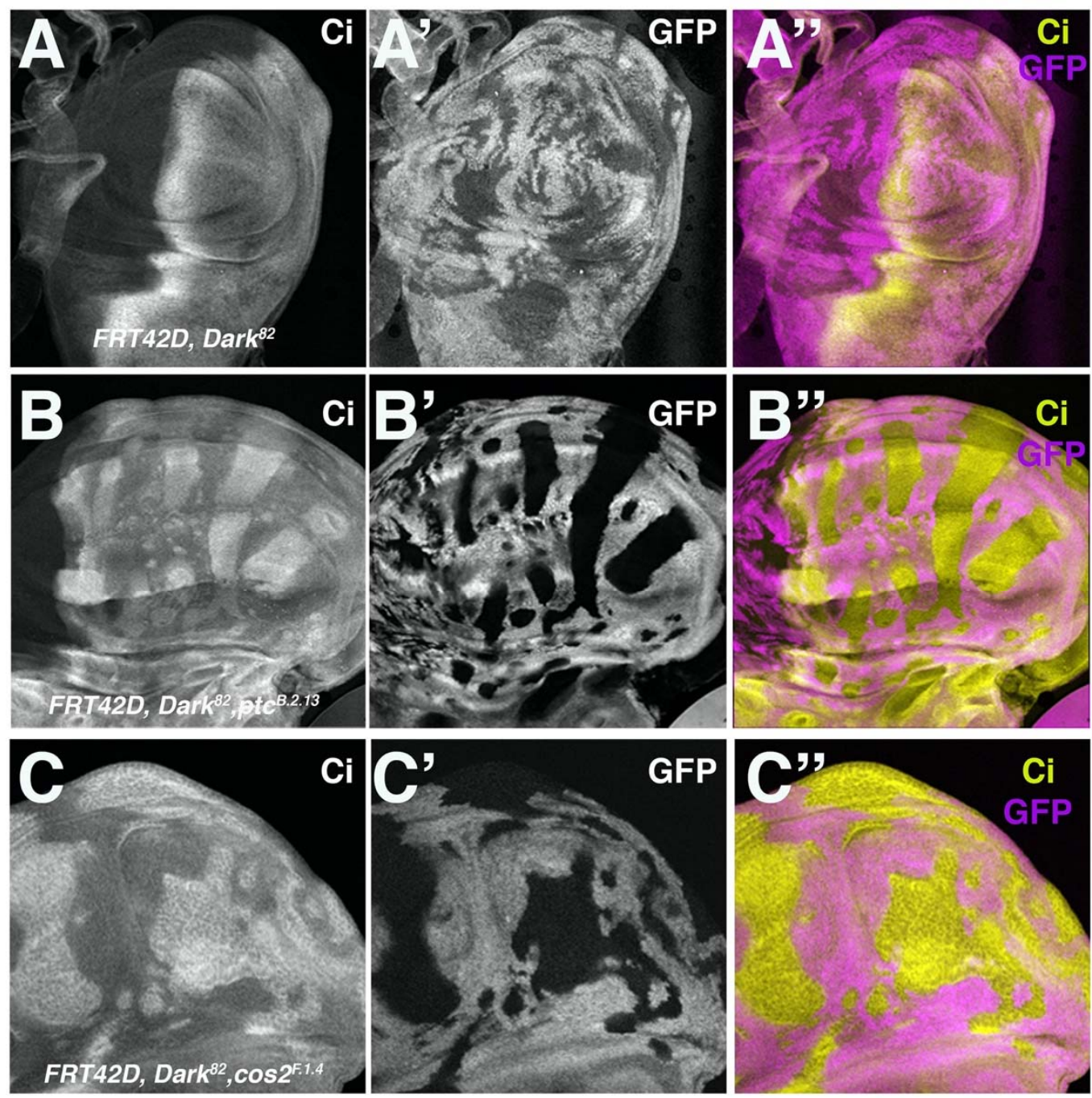

Figure 4: $P t c^{B .2 .13}$ and $\operatorname{Cos} 2^{\text {F.1.4 }}$ autonomously up-regulated $\mathrm{Ci}$ within mutant clones in the anterior compartment of imaginal wing dises

Ci levels visualized in third instar imaginal wing discs through staining and fluorescent microscopy for crosses of UBX-Flp:FRT42D, ubi-GFP mated to (A) control FRT42D, $\operatorname{Dark}^{82}$ (B) FRT42D, tc $^{B .2 .13}, \operatorname{Dark}^{82}$ (C) FRT42D, $\operatorname{Cos} 2^{\text {F.1.4 }}$, $\operatorname{Dark}^{82}$ (mutant tissue is GFP negative). Anterior portion of wing discs are oriented to the right. 


\section{Figure 5}
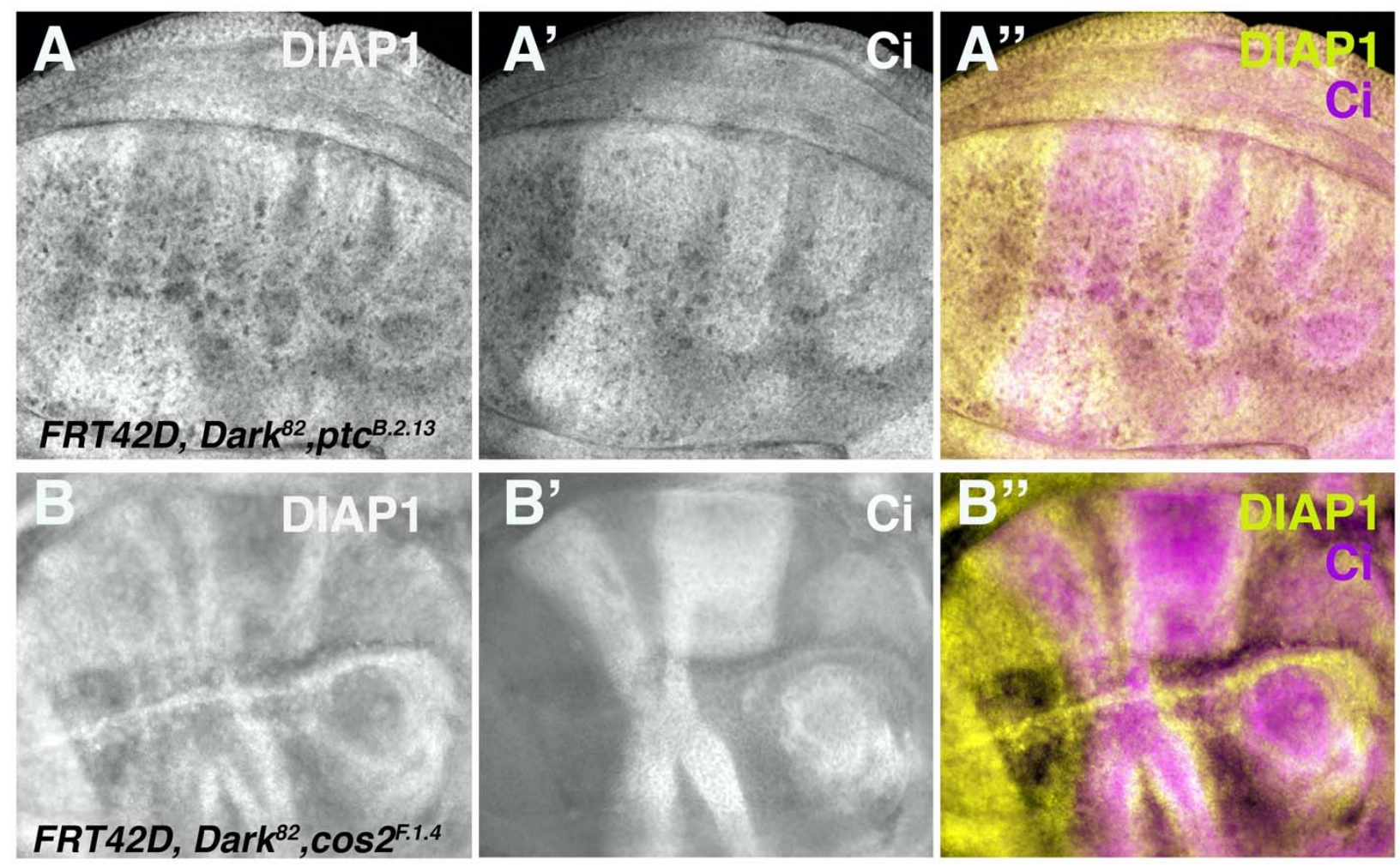

Figure 5: $P t c^{B .2 .13}$ and $\operatorname{Cos} 2^{F .1 .4}$ non-autonomously up-regulated DIAP1 just outside of mutant clone boundaries in the anterior compartment of imaginal wing discs

DIAP1 levels visualized in third instar imaginal wing discs through staining and fluorescent microscopy for crosses of UBX-Flp:FRT42D, ubi-GFP mated to (A) FRT42D, $p t c^{B .2 .13}, \operatorname{Dark}^{82}$ (B) FRT42D, $\operatorname{Cos}^{F .1 .4}$, Dark ${ }^{82}$ (mutant tissue are marked by $\mathrm{Ci}$ up-regulated tissue). Anterior portion of wing discs are oriented to the right. 


\section{Figure 6}
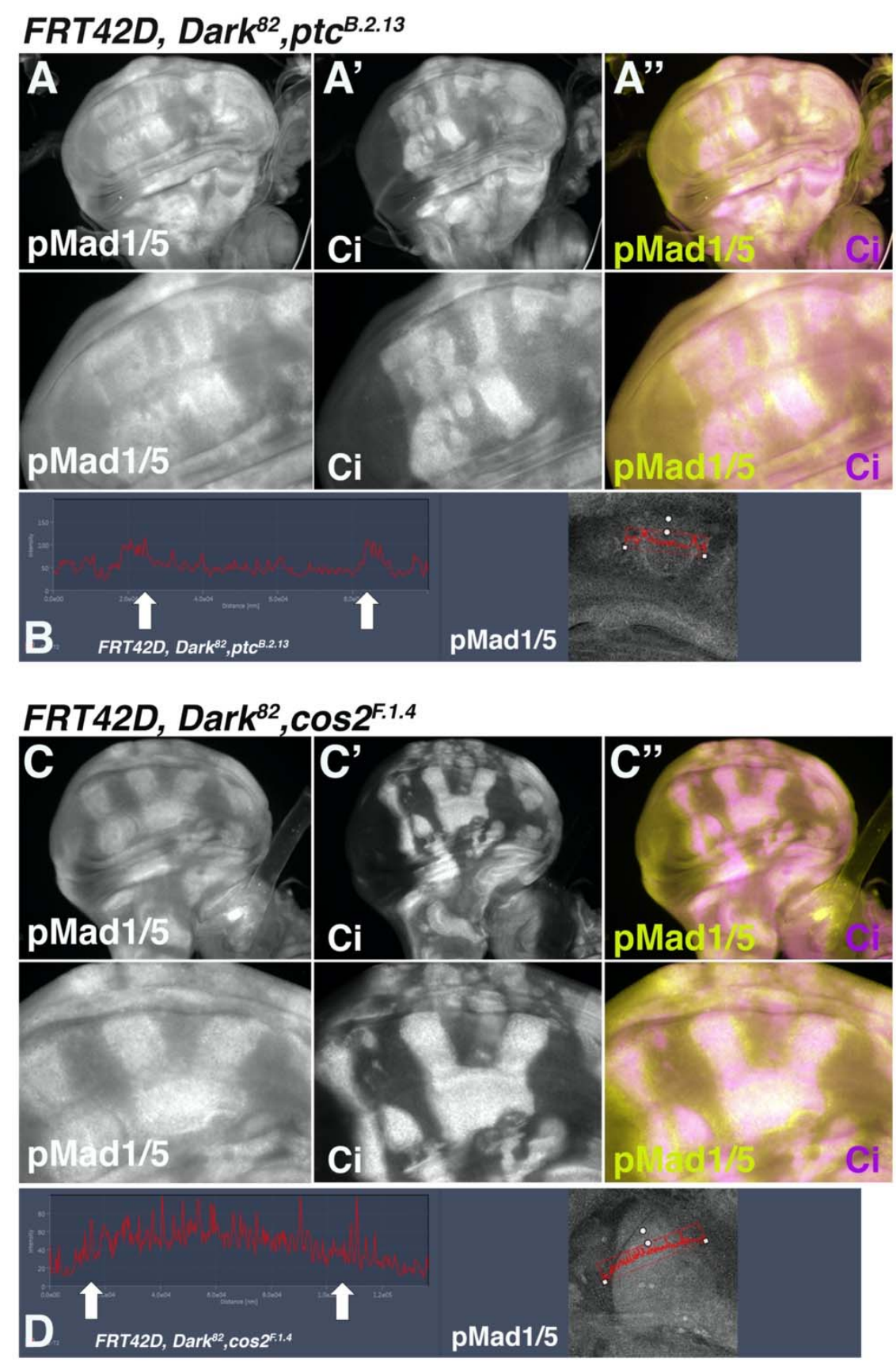

Figure 6: $P t c^{B .2 .13}$ and $\operatorname{Cos} 2^{F .1 .4}$ differentially up-regulate pMad1/5 inside of mutant clones

pMad 1/5 levels visualized in third instar imaginal wing discs through staining and fluorescent microscopy for crosses of UBX-Flp:FRT42D, ubi-GFP mated to (A-A") FRT42D, Ptc ${ }^{B .213}$, Dark ${ }^{82}$ (mutant tissue are marked by Ci up-regulated tissue) (B) quantification of pMad1/5 levels across the mutant clonal boundaries in $P t c^{\text {B.2.13 }}$ mutant clones. Arrows denote clonal boundaries. (C-C') FRT42D, $\operatorname{Cos}^{F .1 .4}, \operatorname{Dark}^{82}$ (mutant tissue are marked by Ci upregulated tissue) (D) quantification of pMad1/5 levels across the mutant clonal boundaries in Ptc ${ }^{B .2 .13}$ mutant clones. Arrows denote clonal boundaries. Anterior portion of wing discs are oriented to the right. 


\section{Supplemental Figure 1}

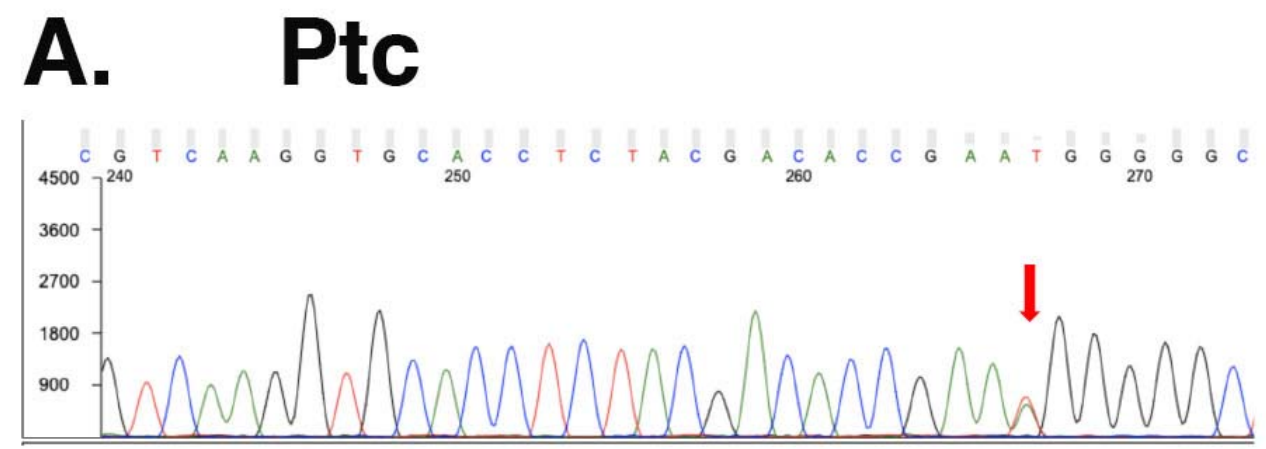

\section{R:8660339 T_A}

\section{Trp-173-Arg}

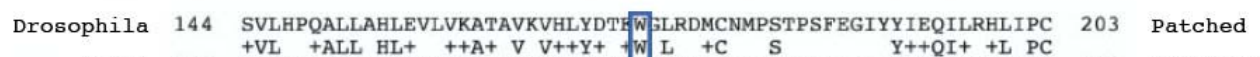
$\begin{array}{lllrlll}\text { Human } & 168 & \text { NVLTTEALLOHLDSALQASRVHVYMYNR WWLEHLC-YKSGELITETGYMDQIIEYLYPC } & 226 & \text { PATCHED-1 }\end{array}$

\section{B. Cos2}

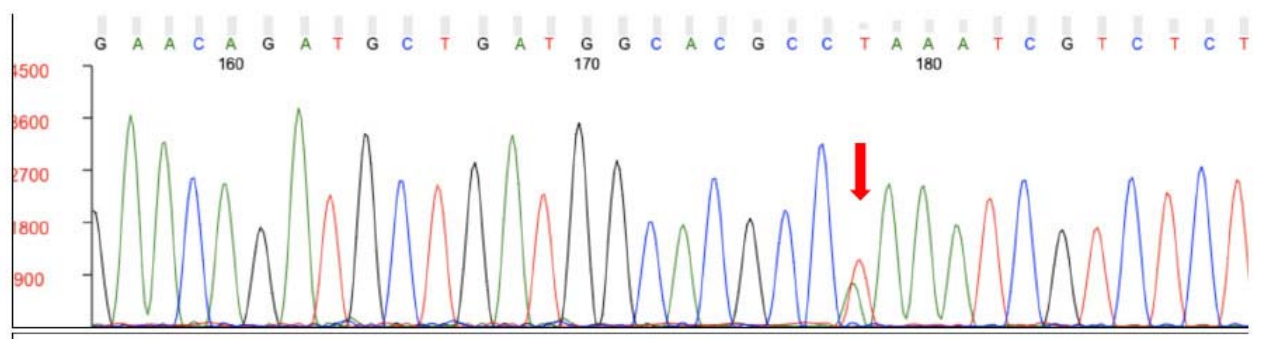

\section{R:7383951 T_A Leu-951-GIn}

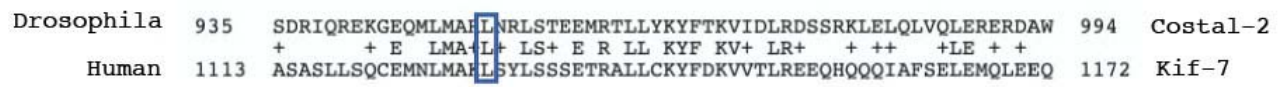

Supplemental Figure 1: $P t c^{B .2 .13}$ and $\operatorname{Cos} 2^{F .1 .4}$ have missense mutations in conserved amino acids

$P t c$ and $\operatorname{Cos} 2$ heterozygous animals were sequenced via Sanger sequencing. (A) Ptc ${ }^{\text {B.2.13 }}$ mutation resides at 2R:8660339 resulting in a Trp-Arg mutation that is in a residue conserved between Patched and PATCHED1. (B). $\operatorname{Cos} 2^{\text {F.1.4 }}$ mutation resides at 2R: 7383951 resulting in a Leu-Gln mutation in a conserved residue between Costal2 and Kif7. 


\section{Supplemental Figure 2}

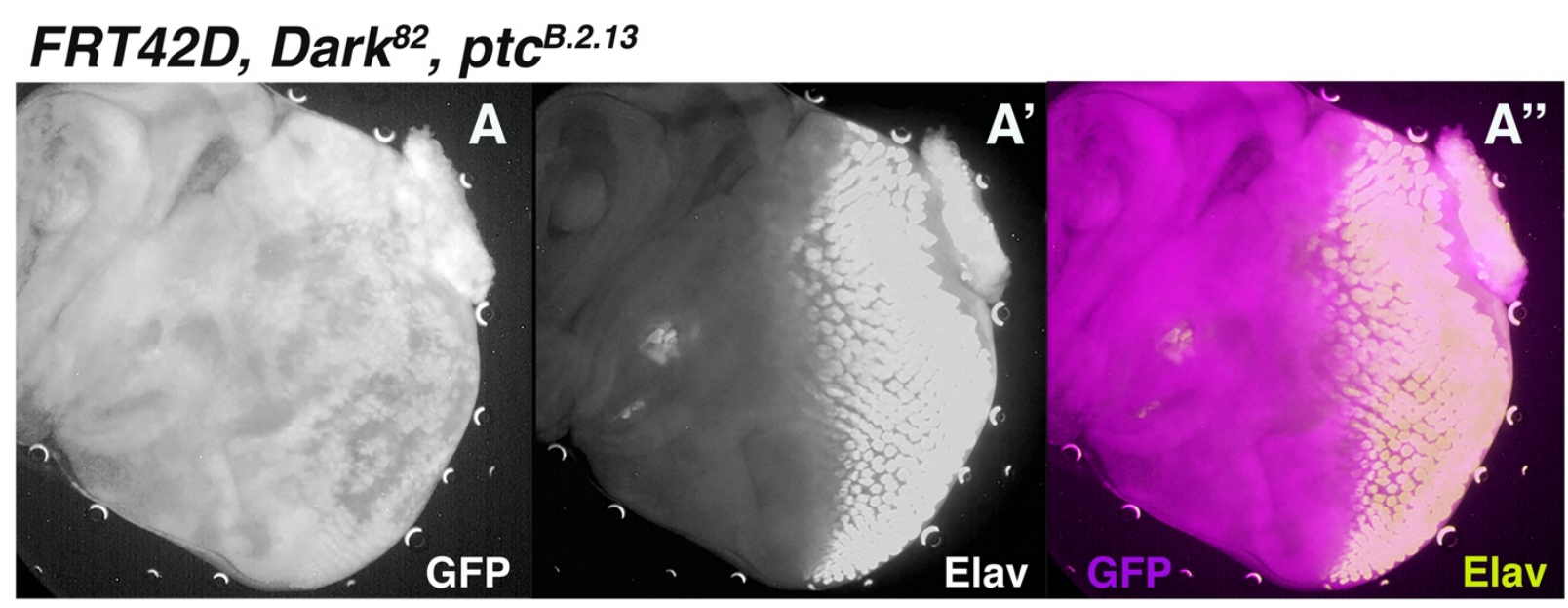

\section{FRT42D, Dark ${ }^{82}, \cos ^{F .1 .4}$}

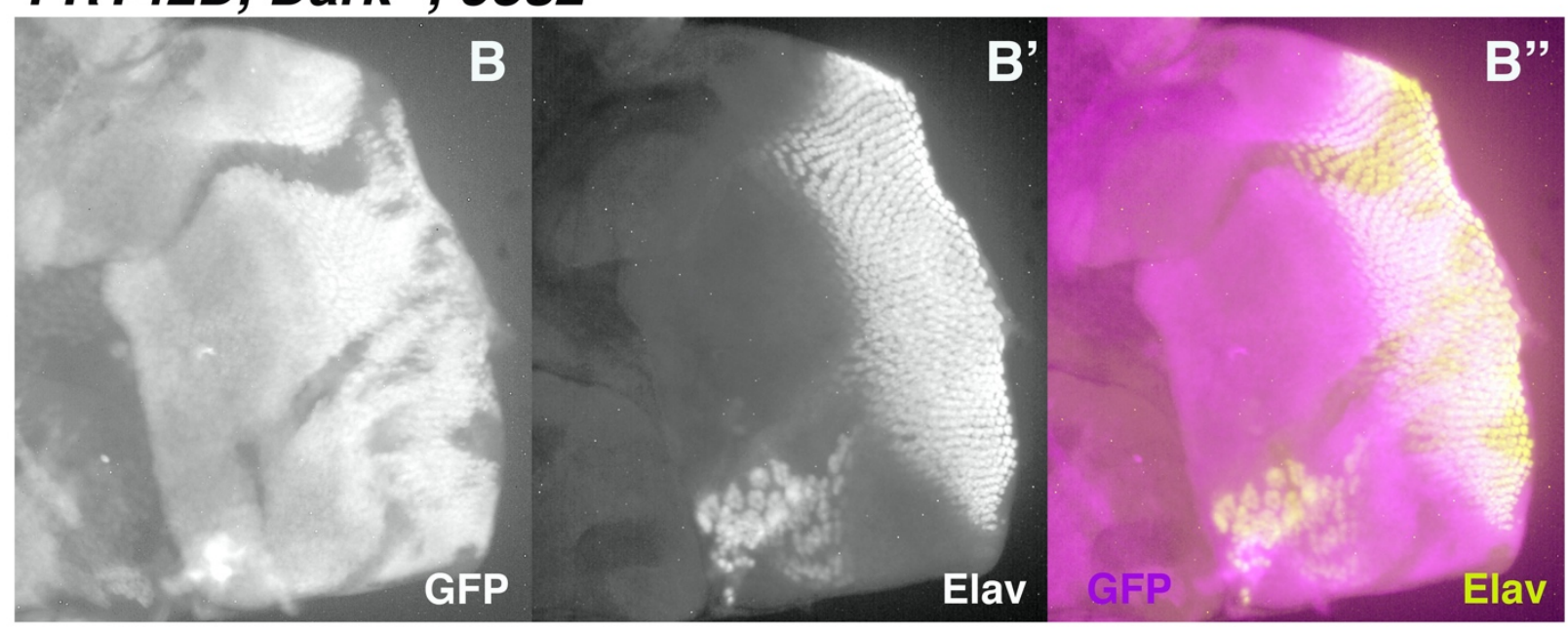

Supplemental Figure 2: Ptc ${ }^{B .2 .13}$ and $\operatorname{Cos} 2^{F .1 .4}$ clones result in premature Elav expression and eye differentiation.

Elav levels visualized in third instar imaginal eye discs through staining and fluorescent microscopy for crosses of Ey-Flp:FRT42D, ubi-GFP mated to (A-A"') FRT42D, Ptc ${ }^{\text {B.2.13 }}$, Dark ${ }^{82}$ and (B-B") FRT42D, $\operatorname{Cos} 2^{F .1 .4}$, Dark $^{82}$ (mutant tissue is GFP negative). In both mutants ectopic Elav expression can be seen in mutant clones prior to the wave of differentiation seen in the rest of the eye. 


\section{Supplemental Figure 3}
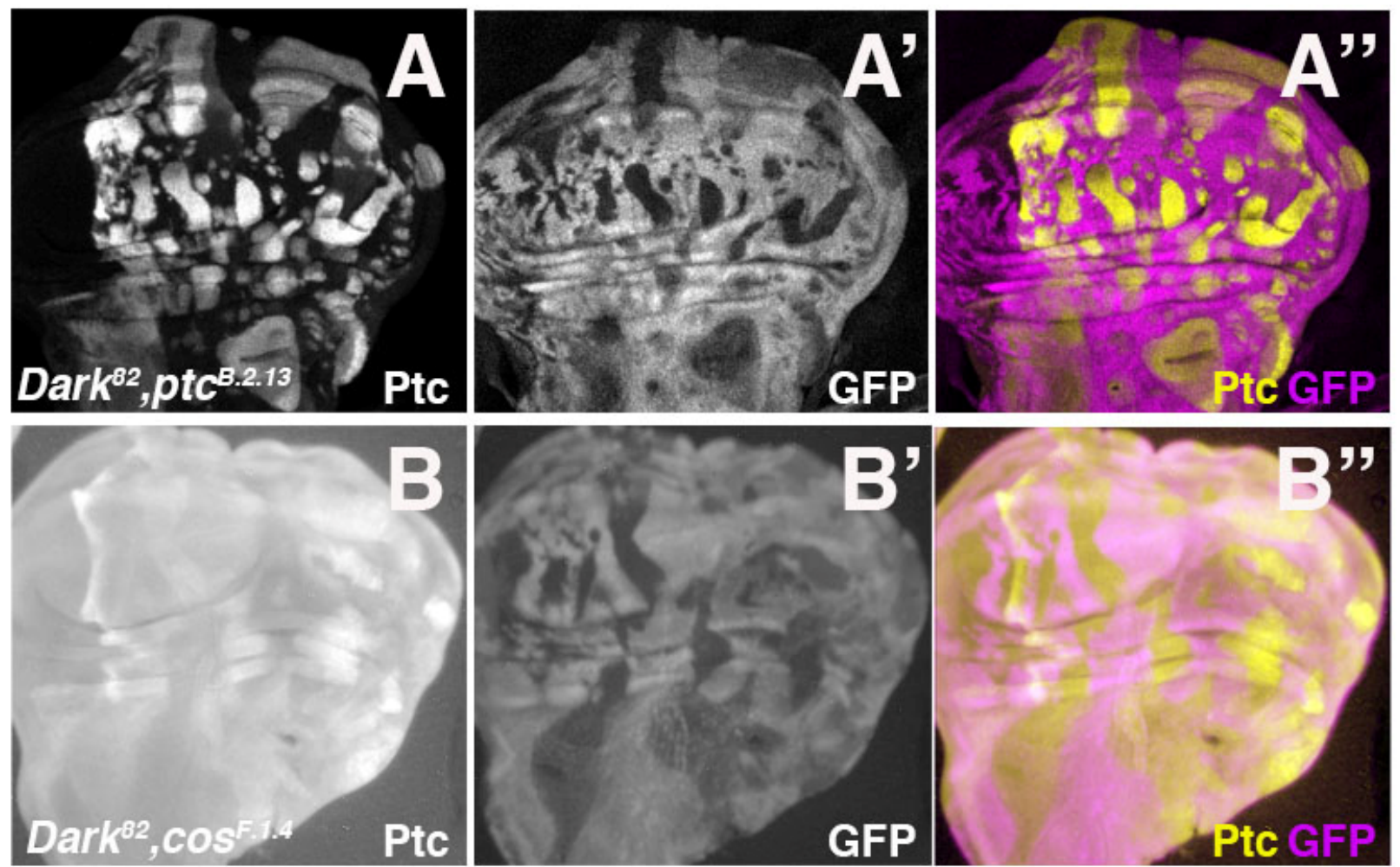

Supplemental Figure 3: $P t c^{B .2 .13}$ and $\operatorname{Cos} 2^{F .1 .4}$ autonomously up-regulated Ptc within mutant clones in the anterior compartment of imaginal wing discs

Ptc levels visualized in third instar imaginal wing discs through staining and fluorescent microscopy for crosses of UBX-Flp:FRT42D, ubi>GFP mated (A-A') FRT42D, Ptc ${ }^{B .2 .13}$, $\operatorname{Dark}^{82}$ and (B-B'") FRT42D, $\operatorname{Cos}^{\text {F.1.4 }}$, Dark ${ }^{82}$ (mutant tissue is GFP negative). Anterior portion of wing discs are oriented to the right. 